\title{
Encoding the unnatural
}

Researchers evolved a ribosome that efficiently decodes quadruplet codons, thus opening up the possibility of genetically incorporating multiple unnatural amino acids into proteins.

Imagine being able to harness the translation system in cells to make genetically encoded polymers for all sorts of useful applications, from materials for tissue engineering to polymer-based therapeutics. Scientists may not be too far away from this fascinating prospect. Jason Chin and his colleagues at the Medical Research Council Laboratory of Molecular Biology in Cambridge, UK, recently reported substantial steps toward this ultimate goal.

Though realizing unnatural polymer synthesis in cells will surely be exciting, methods to site-specifically incorporate unnatural amino acids into proteins have already made an impact on biological research. For more than two decades, researchers have been able to do this in bacteria by reassigning a stop codon, which usually signals the termination of protein synthesis, to encode an unnatural amino acid. In addition to the unique codon, one also needs a unique tRNA that will transfer the unnatural amino acid to the growing peptide chain and a corresponding unique aminoacyl-tRNA synthetase to 'charge' the tRNA with the unnatural amino acid. The translational machinery already present in the cell then carries on its usual business of assembling the protein, incorporating the unnatural amino acid when it reads the reassigned stop codon.

Over the past two decades, various research groups have worked out ways to make this process of constructing proteins with amino acids bearing useful labels or defined posttranslational modifications reasonably efficient in bacteria. The methods have been extended to yeast and mammalian cells. Also, active site mutant synthetase-tRNA pairs have been developed to allow the incorporation of nearly 50 different unnatural amino acids into proteins.

However, extending this approach to knit

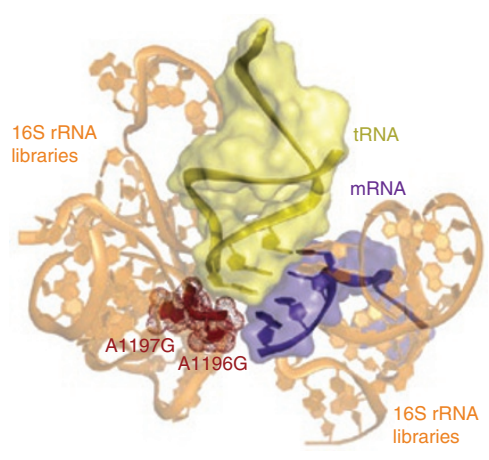

Ribo-Q1 contains two mutations, A1196G and A1197G, which expand the tRNA binding pocket to allow decoding of quadruplet codons with greater efficiency. Reprinted from Nature.

more than one type of unnatural amino acid into a protein has remained a challenge. For one, the number of available codons is fundamentally limited. Of the 64 natural triplet codons, 61 code for the 20 canonical amino acids; only three are stop codons that could potentially be reassigned to encode an unnatural amino acid. To extend the number of 'blank' codons, researchers have previously designed four-base codons; in theory, one could take advantage of all 256 possible blank quadruplet codons to encode 256 different unnatural amino acids. Not surprisingly, though, the endogenous translation machinery does not efficiently decode quadruplet codons.

Chin and his colleagues now report the evolution of an orthogonal ribosome nicknamed ribo-Q1 that can efficiently decode quadruplet codons (Neumann et al., 2010a). Ribo-Q1 operates in a parallel but independent pathway to the natural ribosome, explains Chin. "This allows you to change its properties without interfering with the way that natural translation works," he says. Ribo-Q1 could theoretically allow researchers to encode more than 200 different unnatural amino acids into a protein.

As a proof of principle, Chin and his colleagues demonstrated the incorporation of two chemically reactive unnatural amino acids into defined sites in calmodulin, with high efficiency and fidelity, using ribo-Q1 and two orthogonal synthetase-tRNA pairs. The azide- and alkyne-bearing amino acids react to form a cross-link, constraining the protein structure in a locked conformation. The method could also be used to install probes for imaging and biophysical studies, and to introduce post-translational modifications at specific sites to study their functions. "Variants of each of these [two synthetase-tRNA] pairs have been used to incorporate many amino acids, so there are a large number of possible combinations of amino acids," notes Chin.

To extend the approach to incorporate more than two different unnatural amino acids, however, another aspect of the method needs development. So far only two orthogonal synthetase-tRNA pairs, the tyrosyl-tRNA synthetase-tRNA pair from Methanococcus jannaschii and the pyrrolysyl-tRNA synthetase-tRNA pair from Methanosarcina barkeri, have been evolved at their active sites to charge different unnatural amino acids. Many more unique synthetases that specifically aminoacylate only a single tRNA are needed before incorporating multiple unnatural amino acids into proteins can become a reality. Chin and his colleagues are also pursuing this; they recently described an approach to evolve mutually orthogonal pairs from a single orthogonal pair (Neumann et al., 2010b).

To synthesize unnatural polymers in cells, "the challenge now is to hook up all the bits to go from 2 to $n$," says Chin. "I think we've got an experimental proof that all the steps are feasible, and now we're trying to put together a further layer of complexity."

\section{Allison Doerr}

\section{RESEARCH PAPERS}

Neumann, H. et al. Encoding multiple unnatural amino acids via evolution of a quadruplet-decoding ribosome. Nature 464, 441-444 (2010a).

Neumann, H. et al. De novo generation of mutually orthogonal aminoacyl-tRNA synthetase/tRNA pairs. J. Am. Chem. Soc. 132, 2142-2144 (2010b). 